\title{
Grand Challenges in Environmental Nanotechnology
}

\author{
Hongqi Sun* \\ School of Engineering, Edith Cowan University, Perth, WA, Australia
}

Keywords: environment, nanotechnology, nanomaterials, pollution, remediation, sensing

\section{OVERVIEW}

Energy and environment mark the two key challenges to human beings for the future sustainability of the planet. Modern technologies have been extremely successful in providing renewable energies and conducting environmental remediation. Then the interactions among energy, environment, and technology push the society moving forward to further civilization. However, such paves are also making the environmental issues severer and more complicated.

Environmental sustainability concerns the global climate change and pollutions, e.g., air, soil, and water. Up to now, climate change is still controversial even in major developed countries. Beyond the remit of technology, direct evidences from research, public awareness, and government should play the collaborative role in facing climate change. Meantime, available technologies can offer direct control to environmental pollutions from a variety of sources, for example, heavy metals, organic compounds, biological or radiation hazards, oil spills, and microplastics, etc. Recently, the technologies for environmental applications, for instance, sensing and remediation, have been dramatically advanced by the use of nanomaterials (Khin et al., 2012). This evidenced the emerging and evolving of environmental nanotechnology.

Down to the nanoscale, bulk environmental materials, for example, metals, metal oxides/sulfides, polymers, and carbons, are to possess intriguing physiochemical properties owing to the high surface area-to-volume ratio. Therefore, versatile functionalities and enhanced reactivity/selectivity can be expected as compared to their bulk counterparts. The capacities of

OPEN ACCESS

Edited and reviewed by: Bingqing Wei, University of Delaware, United States

*Correspondence: Hongqi Sun h.sun@ecu.edu.au

Specialty section:

This article was submitted to Environmental Nanotechnology, a section of the journal

Frontiers in Nanotechnology

Received: 09 October 2019 Accepted: 04 December 2019 Published: 20 December 2019

Citation:

Sun H (2019) Grand Challenges in Environmental Nanotechnology. Front. Nanotechnol. 1:2. doi: 10.3389/fnano.2019.00002 environmental nanotechnology can be further enlarged by the manipulation of the nanomaterials in terms of morphology (particle size and shape), microstructure (pore and surface) and composition (heterojunction and doping). New opportunities and challenges then emerge along with the new salient features, e.g., electrical, thermal, optical, magnetic, and electrochemical properties toward adsorptive and/or catalytic performances (Hochella et al., 2019).

\section{INNOVATIVE SOLUTIONS}

Environmental nanotechnology has fundamentally impacted every aspect of the field, ranging from identification to remediation.

(a) Monitoring and sensing: For analysis of heavy metals, inductively coupled plasma mass spectrometry (ICP-MS), atomic absorption/emission spectrometry (AAS/AES), and atomic fluorescence spectroscopy (AFS) can be employed. The monitoring of organic pollutants can be conducted by UV spectroscopy, gas chromatography (GC), high-performance liquid chromatography (HPLC), GC-MS and LC-MS. The combination of the available analytic techniques can monitor most contaminations in soil, air, and water. However, the analysis requires high-cost instrumentation, sophisticated and tedious operation, and well-trained specialists. With the development of nanomaterials, photoelectrochemical analysis integrating the light response and chemical sensing emerged for feasible chemical and biological monitoring (Shi et al., 2019).Very recently, self-propelled nano/micro motors were also proven 
to be a novel approach for environmental sensing (Parmar et al., 2018). The emerging nanopollution would further provide a platform for developing and testing novel monitoring techniques.

(b) Adsorption and nanosorbents: Adsorption is a very mature technology that has been extensively used in environmental remediation. Activated carbon is one of the most popular adsorbents. For example, 44,708 results were obtained when searching two keywords, e.g., activated carbon and adsorption, in the database of Web of Science in September 2019. The concept of nanomaterials extends the dimension of materials, and has exponentially enriched the adsorbent family by nanosorbents. For the carbons only, fullerene, nanodiamonds, quantum dots, nanotubes, graphene, nanospheres, and mesoporous carbons can be obtained with different dimensions $(0 \mathrm{D}, 1 \mathrm{D}, 2 \mathrm{D}$, and $3 \mathrm{D})$. With the functionalization techniques, enormous nanosorbents can be prepared even just based on graphene for various adsorptive applications (Wang et al., 2013).

(c) Membrane and nanomembrane: Membrane technology has been widely employed for gas separation, and water/wastewater treatment. Polymers and ceramics can be used for fabrication of symmetric or asymmetric membranes for traditional separation. Very recently, the studies on nanomaterials led to the fabrication of membrane with nanoscale thickness, namely nanomembranes (Rogers et al., 2011). Such unique membranes can greatly extend the applications of membrane for environmental applications. Membrane and nanomembrane also greatly contribute to the development of nanofiltration technology.

(d) Environmental nanocatalysis: Carrying out chemical reactions over nanostructured catalysts defines nanocatalysis, and then environmental nanocatalysis becomes a very important component in environmental nanotechnology. For example, catalysis over nanostructured materials can significantly promote advanced oxidation processes (AOPs). Fenton reaction, producing hydroxyl radicals by ferrous ions and hydrogen peroxide, is powerful for degradation of organics. Its major limitations of narrow $\mathrm{pH}$ range and sludge formation can be overcome by Fenton-like reactions via nanocatalysis (Yao et al., 2016). As an promising to hydroxyl radical, sulfate radical has also demonstrated outstanding performance for degradation of organic pollutants. The activation methods of peroxymonosulafe (PMS) and persulfate (PS) were developed from ultraviolet, heat, and homogeneous catalysis to heterogeneous catalysis over nanostructured catalysts (Sun et al., 2012). It is interesting to see that the development of nanocatalysis not only contributes to enhanced catalysis, but to enlarged knowledge base by new mechanisms (Duan et al., 2018). In addition, photocatalysis, catalytic ozonation, SCR (selective catalytic reduction), and POPs (persistent organic compounds), and VOCs (volatile organic compounds) removal are also important areas that can efficiently utilize nanocatalysis.

\section{FUTURE CHALLENGES}

Though a huge volume of studies on nanomaterials have been conducted and reported, mature manufacture of desired nanomaterials and their safe applications are not always feasible. Besides the general challenges of nanotechnology, the specific challenges for environmental nanotechnology are as below.

(i) Balance among activity, selectivity and stability: High performances, either adsorptive or catalytic efficiencies in environmental applications, are from the high reactivity owing to the delicate surface and microstructure. The selective adsorption and/or degradation would also be critical for specific environmental issues. Moreover, the harsh and hazardous conditions, which are very common in environmental applications, would easily destruct the composition and/or structure of the nanosized catalysts. The stability, which is the key indicator of practical feasibility, will be then questionable. Compared to chemical manufacture and energy sectors, the stability is more important in environmental applications as metal leaching from nanosized metal-based catalysts and/or newly formed intermediates can become secondary contaminations (Saputra et al., 2013). Rational design of nanomaterials and precise control of the applications should take all the activity, selectivity, and stability into account.

(ii) Mechanistic studies: The new environmental applications driven by nanomaterials would not just demonstrate enhanced performances, but induce different processes which are of unique mechanisms (Duan et al., 2018). The new mechanisms can be originated from the size, shape, composition, surface feature, or hybridizations of the materials. The competitive adsorption or catalysis on the nanomaterials can be also governed by the nature of the materials. Both the environmental processes and materials science should be illustrated to optimize the environmental nanotechnology. Traditional quenching experiments, in situ spectroscopy, and computational studies and their combination are necessary. Chemical engineering efforts might also be essential to apply the theoretical findings for the practical environmental applications.

(iii) Nanopollution and nano/bio interfaces: In most cases opportunity and challenge show up together like twins, which applies to the rise of nanomaterials (Hochella et al., 2019). Nanomaterials and nanotechnologies have enabled extensive biological applications, e.g., sensing, diagnosing, and healing, which can never be realized by bulk materials. Meanwhile, the implications of these applications should be the fate and transport, human and environmental exposure, and direct hazards to human bodies. Nanopollution is no longer a debate, but a real risk. The fundamental understanding, identification, and manipulation at the nano/bio interfaces are then becoming the great challenges of environmental nanotechnology. 


\section{CONCLUSIONS}

Great efforts should be made to monitor and remedy environmental pollutions. The development of nanomaterials and the corresponding nanotechnology have brought up enormous opportunities to conventional environmental technologies. This leads to the rise of environmental nanotechnology. A variety of successful applications, e.g., novel sensing and monitoring, selective adsorption on nanosorbents, nanomembrane separation, and environmental nanocatalysis have been demonstrated. However, there are still great challenges to these pressing issues. Rational design and better utilization of nanomaterials for the environmental processes should find the delicate balance among activity, reactivity, and stability. Mechanistic studies on both the nanotechnology and the environmental processes should be carried out. The hazards of

\section{REFERENCES}

Duan, X. G., Sun, H. Q., and Wang, S. B. (2018). Metal-free carbocatalysis in advanced oxidation reactions. Acc. Chem. Res. 51, 678-687. doi: 10.1021/acs.accounts.7b00535

Hochella, M. F., Mogk, D. W., Ranville, J., Allen, I. C., Luther, G. W., Marr, L. C., et al. (2019). Natural, incidental, and engineered nanomaterials and their impacts on the Earth system. Science 363:eaau8299. doi: 10.1126/science.aau 8299

Khin, M. M., Nair, A. S., Babu, V. J., Murugan, R., and Ramakrishna, S. (2012). A review on nanomaterials for environmental remediation. Energy Environ. Sci. 5, 8075-8109. doi: 10.1039/c2ee2 $1818 \mathrm{f}$

Parmar, J., Vilela, D., Villa, K., Wang, J., and Sanchez, S. (2018). Micro- and nanomotors as active environmental microcleaners and sensors. J. Am. Chem. Soc. 140, 9317-9331. doi: 10.1021/jacs.8b 05762

Rogers, J. A., Lagally, M. G., and Nuzzo, R. G. (2011). Synthesis, assembly and applications of semiconductor nanomembranes. Nature 477, 45-53. doi: 10.1038/nature10381

Saputra, E., Muhammad, S., Sun, H. Q., Ang, H. M., Tade, M. O., and Wang, S. B. (2013). Different crystallographic one-dimensional $\mathrm{MnO}_{2}$ nanomaterials and their superior performance in catalytic phenol degradation. Environ. Sci. Technol. 47, 5882-5887. doi: 10.1021/es4 $00878 \mathrm{c}$ nanomaterials to the environment should also been thoroughly investigated by unraveling the processes at the nano/bio interfaces. The engagement and endeavors to environmental nanotechnology would contribute to future sustainability as a whole.

\section{AUTHOR CONTRIBUTIONS}

The author confirms being the sole contributor of this work and has approved it for publication.

\section{ACKNOWLEDGMENTS}

The author acknowledges the support from the ECU ViceChancellor's Professorial Research Fellowship.

Shi, L., Yin, Y., Zhang, L. C., Wang, S. B., Sillanpaa, M., and Sun, H. Q. (2019). Design and engineering heterojunctions for the photoelectrochemical monitoring of environmental pollutants: a review. Appl. Catal. B Environ. 248, 405-422. doi: 10.1016/j.apcatb.2019.02.044

Sun, H. Q., Liu, S. Z., Zhou, G. L., Ang, H. M., Tade, M. O., and Wang, S. B. (2012) Reduced graphene oxide for catalytic oxidation of aqueous organic pollutants. Acs Appl. Mater. Interfaces 4, 5466-5471. doi: 10.1021/am301372d

Wang, S. B., Sun, H. Q., Ang, H. M., and Tade, M. O. (2013). Adsorptive remediation of environmental pollutants using novel graphene-based nanomaterials. Chem. Eng. J. 226, 336-347. doi: 10.1016/j.cej.2013.04.070

Yao, Y. J., Chen, H., Lian, C., Wei, F. Y., Zhang, D. W., Wu, G. D., et al. (2016). $\mathrm{Fe}, \mathrm{Co}$, Ni nanocrystals encapsulated in nitrogen-doped carbon nanotubes as Fenton-like catalysts for organic pollutant removal. J. Hazard. Mater. 314, 129-139. doi: 10.1016/j.jhazmat.2016.03.089

Conflict of Interest: The author declares that the research was conducted in the absence of any commercial or financial relationships that could be construed as a potential conflict of interest.

Copyright (C) 2019 Sun. This is an open-access article distributed under the terms of the Creative Commons Attribution License (CC BY). The use, distribution or reproduction in other forums is permitted, provided the original author(s) and the copyright owner(s) are credited and that the original publication in this journal is cited, in accordance with accepted academic practice. No use, distribution or reproduction is permitted which does not comply with these terms. 